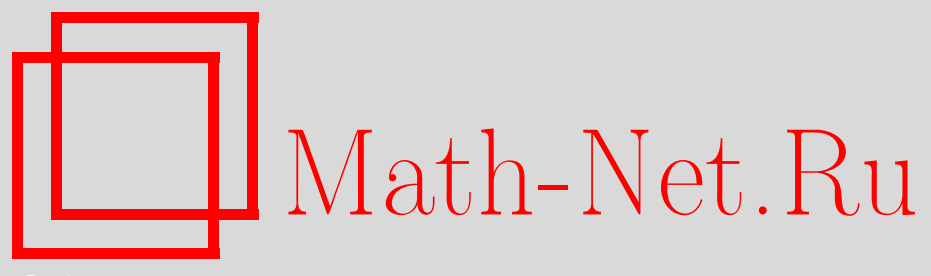

Д. А. Бусарова, Робастное оценивание матрицы коэффициентов в многомерной линейной регрессионной модели, УМH, 2006, том 61, выпуск 3, 169-170

DOI: https://doi.org/10.4213/rm1746

Использование Общероссийского математического портала Math-Net.Ru подразумевает, что вы прочитали и согласны с пользовательским соглашением http://www.mathnet.ru/rus/agreement

Параметры загрузки:

IP: 54.147 .182 .235

26 апреля 2023 г., 16:26:00

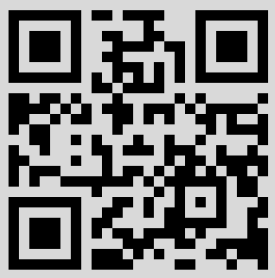




\section{Робастное оценивание матрицы коэффициентов в многомерной линейной регрессионной модели}

\section{Д. А. Бусарова}

Рассмотрим модель многомерной линейной регрессии:

$$
Y_{i}=B_{0}^{\mathrm{T}} X_{i}+\varepsilon_{i}, \quad i=1, \ldots, n,
$$

где $Y_{i}$ - наблюдаемый $(q \times 1)$-вектор отклика, $X_{i}$ - наблюдаемый $(p \times 1)$-вектор фактора и $\varepsilon_{i}-(q \times 1)$-вектор случайных ошибок, $\varepsilon_{i} \stackrel{d}{=}-\varepsilon_{i}$. Мы будем предполагать, что как фактор $X_{i}$, так и отклик $Y_{i}$ являются случайными. Наша задача - по независимым наблюдениям $\left(X_{i}, Y_{i}\right), i=1, \ldots, n$, оценить неизвестную $(p \times q)$-матрицу коэффициентов $B_{0}$.

Непараметрическая оценка, представленная в статье, робастна и аффинно-эквивариантна. Она базируется на понятиях элементарных множеств и элементарных регрессий. Каждое элементарное множество есть подмножество $I=\left\{i_{1}, \ldots, i_{p}\right\}$ размера $p$ исходных наблюдений. Далее, пусть $Y(I)$ есть $(p \times q)$-матрица $\left(Y_{i_{1}}, \ldots, Y_{i_{p}}\right)^{\mathrm{T}}$ и $X(I)-(p \times p)$-матрица $\left(X_{i_{1}}, \ldots, X_{i_{p}}\right)^{\mathrm{T}}$. Если $\operatorname{rank}(X(I))=p$, то элементарная регрессия определяется как $B(I)=X(I)^{-1} Y(I)$. Если же $\operatorname{rank}(X(I))<p$, то элементарная регрессия вырождена. Векторизуем каждую невырожденную элементарную регрессию с помощью операции vес: $b(I)=\operatorname{vec}(B(I))$ (операция vес преобразует $(p \times q)$-матрицу в $p q$-вектор, помещая последовательно столбцы матрицы друг под другом). Обозначим далее $\beta_{0}=\operatorname{vec}\left(B_{0}\right)$. Для аффинно-эквивариантного оценивания вектора $\beta_{0}$ (а значит, и матрицы $B_{0}$ ) будем рассматривать медиану Оя (см. [1]) совокупности векторизованных невырожденных элементарных регрессий $\{b(I)\}$.

Введем обозначения. Пусть $I, I_{1}, I_{2}, \ldots$ обозначают подмножества размера $p$ множества $1, \ldots, n$. Обозначим $\mathscr{I}=\left\{\left\{I_{1}, \ldots, I_{p q}\right\}\right\}$, и пусть

$$
\mathscr{I}_{p}=\left\{\left\{I_{1}, \ldots, I_{p q}\right\} \in \mathscr{I}:\left|I_{1} \cup \cdots \cup I_{p q}\right|=p^{2} q\right\} .
$$

Назовем элемент $\left\{I_{1}, \ldots, I_{p q}\right\}$ вырожденным, если хотя бы одно из входящих в него элементарных подмножеств вырождено. Пусть функция $\tau\left(I_{1}, \ldots, I_{p q}\right)$ является индикатором этого; она равна 0, если элемент $\left\{I_{1}, \ldots, I_{p q}\right\}$ вырожден, и 1 иначе. Причем в вырожденном случае для любой функции $f\left(I_{1}, \ldots, I_{p q}\right)$, принимающей значения из $\mathbb{R} \cup\{+\infty\} \cup\{-\infty\}$, будем считать, что $f\left(I_{1}, \ldots, I_{p q}\right) \cdot \tau\left(I_{1}, \ldots, I_{p q}\right)=0$.

Рассмотрим целевую функцию:

$$
U_{n}(\beta)=\frac{1}{\left|\mathscr{I}_{p}\right|} \sum_{\mathscr{I}_{p}} V\left(\beta, b\left(I_{1}\right), \ldots, b\left(I_{p q}\right)\right) \tau\left(I_{1}, \ldots, I_{p q}\right),
$$

где $V\left(\varepsilon_{1}, \ldots, \varepsilon_{k+1}\right)$ обозначает объем $k$-мерного симплекса с вершинами $\varepsilon_{1}, \ldots, \varepsilon_{k+1} \in$ $\mathbb{R}^{k}$.

ОпредЕлЕниЕ. Оценку $\widehat{\beta}_{n}$ вектора коэффициентов $\beta_{0}$ определим следующей формулой:

$$
\widehat{\beta}_{n}=\arg \min _{\beta \in \mathbb{R}^{p q}} U_{n}(\beta) .
$$

Разложив определитель по первому столбцу, определим:

$$
\left|d_{0}\left(I_{1}, \ldots, I_{p q}\right)+d^{\mathrm{T}}\left(I_{1}, \ldots, I_{p q}\right) \beta\right|=\frac{1}{(p q) !}\left|\operatorname{det}\left(\begin{array}{cccc}
1 & 1 & \ldots & 1 \\
\beta & b\left(I_{1}\right) & \ldots & b\left(I_{p q}\right)
\end{array}\right)\right| \tau\left(I_{1}, \ldots, I_{p q}\right) .
$$

Пусть, далее, $Z_{i}=\left(X_{i}^{\mathrm{T}}, Y_{i}^{\mathrm{T}}\right)^{\mathrm{T}}$ и $I_{k}=((k-1) p+1, \ldots, k p), k=1, \ldots, p q$. Определим функцию

$$
U(\beta)=\mathrm{E}\left|d_{0}\left(I_{1}, \ldots, I_{p q}\right)+d^{\mathrm{T}}\left(I_{1}, \ldots, I_{p q}\right) \beta\right|,
$$


случайный вектор

$$
\Lambda\left(Z_{1}\right)=\mathrm{E}\left(\operatorname{sign}\left(d_{0}\left(I_{1}, \ldots, I_{p q}\right)+d^{\mathrm{T}}\left(I_{1}, \ldots, I_{p q}\right) \beta_{0}\right) d\left(I_{1}, \ldots, I_{p q}\right) \mid Z_{1}\right),
$$

векторную функцию

$$
A(T, Z)=\mathrm{E}\left(\operatorname{sign}\left(d_{0}\left(I_{1}, \ldots, I_{p q}\right)+d^{\mathrm{T}}\left(I_{1}, \ldots, I_{p q}\right) T\right) d\left(I_{1}, \ldots, I_{p q}\right) \mid Z_{1}=Z\right),
$$

где $T \in \mathbb{R}^{p q}$ и $Z \in \mathbb{R}^{p+q}$, и матрицу $\Gamma=\operatorname{cov}\left(\Lambda\left(Z_{1}\right), \Lambda\left(Z_{1}\right)\right)$. Оценка vec ${ }^{-1}\left(\widehat{\beta}_{n}\right)$ аффинноэквивариантна, а также обладает следующими свойствами:

Теорема 1. Пусть выполнены следующие условия:

(а) $\varepsilon_{1}, \ldots, \varepsilon_{n}$ - независимые одинаково распределенные случайные величины, $\varepsilon_{1} \stackrel{d}{=}-\varepsilon_{1} ; X_{1}, \ldots, X_{n}$ - независимые одинаково распределенные случайные величины; совокупности $\left\{X_{i}\right\}_{i=1}^{n} u\left\{\varepsilon_{i}\right\}_{i=1}^{n}$ независимы;

(b) $\mathrm{E}\left\|\varepsilon_{1}\right\|^{2}<\infty$;

(c) $\mathrm{E}\left(\left\|X(I)^{-1}\right\|^{2} I(\operatorname{det} X(I) \neq 0)\right)<\infty$;

(d) функиия $U(\beta)$ достигает минимум в единственной точке $\beta=\beta^{*}$;

(e) $\left.\nabla U(\beta)\right|_{\beta=\beta^{*}}=\mathrm{E}\left(\nabla\left|d_{0}\left(I_{1}, \ldots, I_{p q}\right)+d^{\mathrm{T}}\left(I_{1}, \ldots, I_{p q}\right) \beta\right|_{\beta=\beta^{*}}\right)$;

(f) в окрестности точки $\beta^{*}$ верно следующее разложение:

$$
U(\beta)=U\left(\beta^{*}\right)+\frac{1}{2}\left(\beta-\beta^{*}\right)^{\mathrm{T}} W\left(\beta-\beta^{*}\right)+o\left(\left\|\beta-\beta^{*}\right\|^{2}\right),
$$

где $W$ - положительно определенная матрица;

(g) $n p u\left\{I_{1}, \ldots, I_{p q}\right\} \in T_{p, p q}(n)$

$$
\mathrm{P}\left(\left\{\operatorname{det}\left(b\left(I_{1}\right)-\beta^{*}, \ldots, b\left(I_{p q}\right)-\beta^{*}\right)=0\right\} \cap\left\{\operatorname{det} X\left(I_{1}\right) \cdots \operatorname{det} X\left(I_{p q}\right) \neq 0\right\}\right)=0 .
$$

Тогда $\sqrt{n}\left(\widehat{\beta}_{n}-\beta_{0}\right) \stackrel{d}{\rightarrow} N_{p q}\left(0, p^{4} q^{2} W^{-1} \Gamma W^{-1}\right)$ nрu $n \rightarrow \infty$.

Теорема 2. Пусть выполнены условия (a), (b), (c), (d), (f) теоремы 1, а также

$\left(\mathrm{e}^{\prime}\right)$ для всех $\beta^{\prime}$ из некоторой окрестности точки $\beta^{*}$ выполнено

$$
\left.\nabla U(\beta)\right|_{\beta^{\prime}}=\mathrm{E}\left(\left.\nabla\left|d_{0}\left(I_{1}, \ldots, I_{p q}\right)+d^{\mathrm{T}}\left(I_{1}, \ldots, I_{p q}\right) \beta\right|\right|_{\beta^{\prime}}\right) ;
$$

(h) функция $A(T, Z)$ непрерывна по $T$ в окрестности точки $T=\beta^{*}$.

Тогда функция влияния оченки $\widehat{\beta}_{n}$ вектора коэффициентов $\beta_{0}$ ограничена и равна

$$
\operatorname{IF}\left(Z, F_{\beta_{0}}, \widehat{\beta}_{n}\right)=-p^{2} q W^{-1} A\left(\beta_{0}, Z\right) .
$$

ЗАмечАниЕ. Условие (с) теорем сильно ограничивает множество удовлетворяющих ему законов распределения. Поэтому тестовую статистику $\psi_{n}$ целесообразно применять для статистических выводов в условиях активного эксперимента (когда исследователь сам выбирает план эксперимента). Как известно из теории оптимального планирования (см. [2]), в условиях активного эксперимента оптимальными являются планы с конечным числом значений, а такие планы (т.е. распределения $X_{1}$ ) удовлетворяют условию (c).

Автор выражает глубокую благодарность Ю. Н. Тюрину, под руководством которого выполнена эта работа, а также М. В. Болдину и В.Н. Тутубалину за ценные замечания и советы.

\section{Список литературы}

[1] Н. Oja, Statist. Probab. Lett., 1:6 (1983), 327-332. [2] С. М. Ермаков, А. А. Жиилявский, Математическая теория оптимального эксперимента, Наука, М., 1987.

Д. А. Бусарова (D. A. Busarova)

Московский государственный университет им. М. В. Ломоносова

E-mail: alekseevad@mail.ru
Представлено А. В. Булинским Принято редколлегией 04.04.2006 